\title{
The Ultra-High-Energy Source MGRO J1908+06
}

\author{
Kelly Malone ${ }^{a, *}$ on behalf of the HAWC Collaboration \\ (a complete list of authors can be found at the end of the proceedings) \\ ${ }^{a}$ Los Alamos National Laboratory, \\ Los Alamos, NM, USA \\ E-mail: kmalone@lanl.gov
}

The TeV gamma-ray source MGRO J1908+06 is one of the highest-energy sources known, with observed emission by the High Altitude Water Cherenkov (HAWC) Observatory extending well past $100 \mathrm{TeV}$. The source exhibits both energy-dependent morphology and a spatially-dependent spectral index. The emission is likely to be dominantly leptonic, and associated with the radioquiet PSR J1907+0602. However, one-population models do not describe the data well; a second particle population is needed to explain the shape of the spectral energy distribution at the highest energies. This component can be well-described by either leptonic or hadronic hypotheses. We discuss this feature and implications for detection by multi-wavelength and multi-messenger experiments.

\footnotetext{
*Presenter
} 


\section{Previous observations}

The ultra-high-energy source MGRO J1908+063 was discovered by the Milagro experiment in 2007 [1] and subsequently observed by H.E.S.S., ARGO, VERITAS, and HAWC, among others [25]. The source is exceptionally bright, spatially extended, and has a hard spectrum with little or no curvature up to $\sim 20 \mathrm{TeV}$. Recent observations by HAWC indicate that the source emits well past $100 \mathrm{TeV}$ [6]. The maximum photon energy is $218 \mathrm{TeV}$ at the $95 \%$ confidence level [7].

The region surrounding the $\mathrm{TeV}$ emission is fairly crowded, with many possible counterparts to the emission. There are two pulsars: PSR J1907+0602 and PSR J1907+0631, as well as SNR G40.5-0.5. PSR J1907+0602 is an exceptionally powerful, radio-faint pulsar, with an $\dot{E}$ of $2.8 \times$ $10^{36} \mathrm{erg} / \mathrm{s}$ [8]. Historically, the $\mathrm{TeV}$ emission has been attributed to the nebula surrounding this pulsar. There are also several molecular clouds in the region [9].

The pulsar wind nebula has not been detected at energies below $\mathrm{GeV}$; for example no extended emission has been observed in the X-ray band. Extended $\mathrm{GeV}$ emission was undetected until recently [10]. An analysis using Fermi-LAT data found that there are likely two components in the $\mathrm{GeV}$ region: a lower-energy $(<10 \mathrm{GeV})$ component associated with the SNR and a higher-energy component attributed to inverse Compton scattering.

Due to the hard spectrum of the $\mathrm{TeV}$ emission, this source has long been considered a potential neutrino source. It has the best p-value for a Galactic source in IceCube catalog searches, although it is still consistent with background [11].

In this proceeding, we investigate the spectrum and morphology of the source using HAWC data. We will then discuss possible origins of the $\mathrm{TeV}$ emission. Note that the results presented here will be submitted for publication soon, with the analysis and results described in much more detail.

\section{HAWC results}

The analysis presented here uses data from the High Altitude Water Cherenkov (HAWC) Gamma-Ray Observatory, which is an extensive air shower array located at an altitude of 4100 meters in the state of Puebla, Mexico. It is optimized to detect gamma rays with energies between a few hundred $\mathrm{GeV}$ and a few hundred TeV. For more details about HAWC, refer to [12].

This analysis uses 1343 days of data with reconstructed energies above $1 \mathrm{TeV}$. The energy is estimated using the "ground parameter" energy estimation method, which relies on the charge measured in the PMTs 40 meters from the shower core along with the zenith angle of the event [13].

The spectrum and morphology are simultaneously determined via a likelihood fit. There are three sources in the model: 3HWC J1908+063 (where 3HWC refers to HAWC's third catalog of sources [5], the most recent available) as well as the lobes of SS433, which are spatially coincident with the edge of the source [14]. A significance map of the region can be seen in Figure 1. The likelihood fit is performed using the HAWC Accelerated Likelihood (HAL) ${ }^{1}$ plugin to the $3 \mathrm{ML}$ (the Multi-mission maximum likelihood) software package [15].

\footnotetext{
${ }^{1}$ https://github.com/threeML/hawc_hal
} 


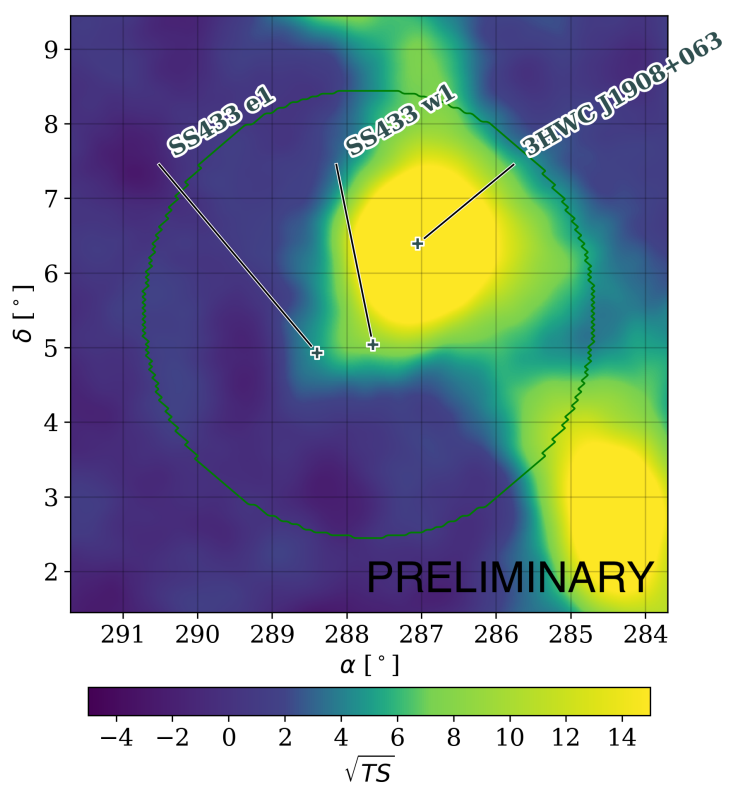

Figure 1: HAWC significance map of the region, for $1<E_{\text {reconstructed }}<316 \mathrm{TeV}$. The green circle denotes the region of interest for the likelihood fit. The three sources included in the model are labeled. The color scale is set to make it easy to see where the $5 \sigma$ threshold is but is saturated at the end of the scale. The maximum significance in the ROI is $38.8 \sigma$.

The best-fit spectrum is a log parabola shape detected in the range from $\sim 500 \mathrm{GeV}$ to $\sim 200 \mathrm{TeV}$. The best-fit morphology is a diffusion morphology where the particles are continuously injected from the center of the source. The details of the diffusion model can be found in [16].

\subsection{Morphology studies}

The source exhibits energy-dependent morphology, with the size of the source decreasing with energy. This could be an indication that the emission is predominantly leptonic, as the higherenergy particles are not able to travel far before cooling. The source remains spatially extended at the highest energies.

The spectral index can also be spatially resolved. If one draws four concentric rings around the center of the source and fits the spectrum to a power-law in each one, a statistically significant softening of the spectrum is observed as a function of distance from the center of the source. This is also an indication of leptonic emission, as electrons far away from the center of the source are likely older and have cooled. No spectral change is expected for hadrons, since the proton cooling timescale is approximately infinitely long.

\subsection{Potential spectrum hardening feature}

A potentially interesting feature can be seen at the highest energies. A standard HAWC analysis contains energy bins that are a quarter-decade wide in log-energy space. When the last three typical energy bins are subdivided into six smaller bins of equal size, an apparent flattening in the spectrum can be seen by eye, with the flux points deviating from the best-fit log parabola spectrum. 
This effect is not presently statistically significant; the amount of the deviation from the best-fit $\log$ parabola is approximately $2 \sigma$. However, if this feature is shown to be significant with either improved reconstruction algorithms or more data, it could be indicative of a second population of particles contributing to the gamma-ray emission at the highest energies.

For a more in-depth discussion of this spectrum hardening feature, see [17] in these proceedings.

\section{The origin of the emission}

Modeling indicates that one-population models are unlikely. Looking at the gas in the region, a one-population hadronic model is difficult to explain as there is not enough energy available to account for the source's observed flux. It is difficult to fit a one-population leptonic model to the observed $\mathrm{TeV}$ shape.

Therefore, two populations of particles are needed to explain the shape of the spectrum in the $\mathrm{TeV}$ range. One potential model consists of a leptonic component that provides an explanation for the bulk of the emission, with a second component that contributes mainly at the highest energies. HAWC's uncertainties are currently too large to determine if this second component is leptonic or hadronic in origin.

The first leptonic component is likely the PWN associated with PSR J1907+0602. The second component could be produced by a variety of different explanations. If it is leptonic, it could originate from the second pulsar in the region, PRS J1907+0631. If hadronic, it could be linked to the supernova remnant. More exotic explanations, such as hadron acceleration in PWN, are also plausible.

Figure 2 shows the HAWC spectrum with potential models highlighted. Each model as two components in the $\mathrm{TeV}$ range: the thick blue line denotes the two-population leptonic model, while the solid red line denotes a lepto-hadronic model. Both models contain an additional component in the $\mathrm{GeV}$ range, based on recent Fermi data that indicates there is a hadronic component below 10 $\mathrm{GeV}$. Since HAWC is not sensitive to this energy range, we simply use the parameters from [10].

\section{Future outlook}

The uncertainties are presently too large to distinguish whether the component prominent above $50 \mathrm{TeV}$ is leptonic or hadronic in origin. The HAWC Collaboration is currently analyzing a new pass over all collected data, with updated reconstruction algorithms and better gamma/hadron separation algorithms. This may allow for the necessary sensitivity at high energies to distinguish between these two components. HAWC's recently-completed outrigger array will also increase the experiment's sensitivity at the highest energies.

This source is also an attractive target for other experiments. LHAASO has recently detected this source above $100 \mathrm{TeV}$ with a significance of $17.2 \sigma$ [18]. Detailed morphology studies above this threshold could help uncover the nature of the source. The two different models presented here start to diverge rapidly just above the energy range that HAWC is sensitive to.

Multi-wavelength data will also be important. The leptonic model and the lepto-hadronic model have very different fluxes in the $\mathrm{keV}$ to $\mathrm{MeV}$ energy bands. In-depth measurements from 


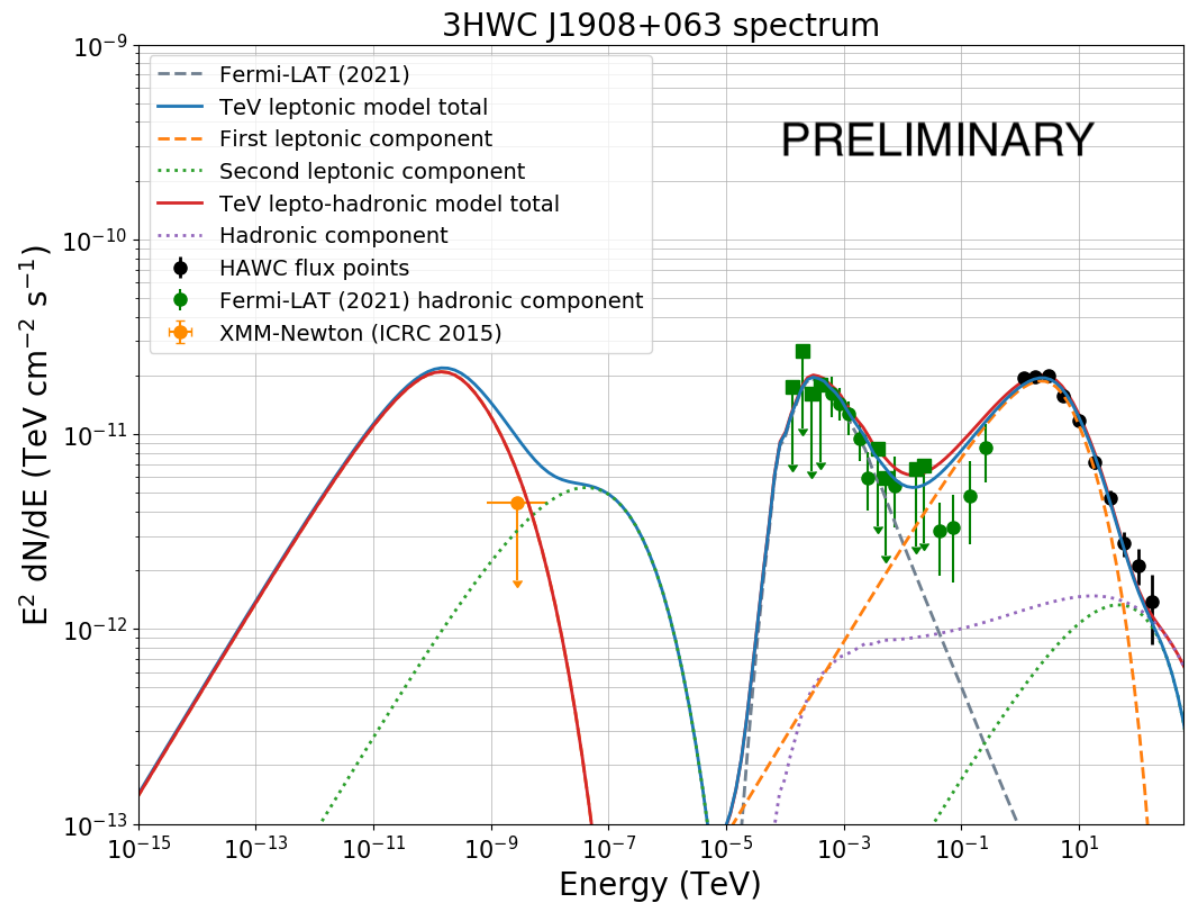

Figure 2: Potential models that fit the available multi-wavelength data. The thick red line consists of the hadronic component around $10 \mathrm{GeV}$ from [10] along with two leptonic components in the $\mathrm{TeV}$ (the sum of the dashed grey + dashed orange + dotted green lines). The thick blue line consists of the hadronic GeV component along with a leptonic component that is prominent in the multi-TeV regime and a second hadronic component at the very highest energies (the sum of the dashed grey + dashed orange + dotted purple lines)

X-ray experiments such as NuSTAR will be useful, as will observations from proposed experiments such as AMEGO [19]. X-ray to $\mathrm{GeV}$ observations will also allow for better determination of the spectral shape and energy budget.

As mentioned in the introduction, this source has long been thought of as a potential neutrino source. Many published predictions of how much data IceCube will need to accumulate before detecting the source assume that it is completely hadronic in nature [20,21]. However, the analysis presented here asserts that the source is dominantly leptonic, with only a fractional hadronic contribution. Those predictions should be re-evaluated in light of this. IceCube-Gen2 may be necessary to detect the source using neutrinos.

\section{Conclusions}

We report on the spectrum and morphology of 3HWC J1908+063, which is one of the highestenergy known gamma ray sources. It is well-fit to a log-parabola spectrum and a diffusive morphological model. There is evidence that the source exhibits energy-dependent morphology, and there may be hints of spectral hardening at the highest energies. More data, both from HAWC's upgraded array and recently-constructed experiments such as LHAASO, are needed to confirm this. 
It is likely that the source emission originates from mutliple populations of particles, with the bulk of the emission being leptonic and powered by the high- $\dot{E}$ pulsar PSR J1907+0602. However, a hadronic contribution at the highest energies cannot be ruled out.

\section{Acknowledgments}

We acknowledge the support from: the US National Science Foundation (NSF); the US Department of Energy Office of High-Energy Physics; the Laboratory Directed Research and Development (LDRD) program of Los Alamos National Laboratory; Consejo Nacional de Ciencia y Tecnología (CONACyT), México, grants 271051, 232656, 260378, 179588, 254964, 258865, 243290, 132197, A1-S-46288, A1-S-22784, cátedras 873, 1563, 341, 323, Red HAWC, México; DGAPA-UNAM grants IG101320, IN111716-3, IN111419, IA102019, IN110621, IN110521; VIEP-BUAP; PIFI 2012, 2013, PROFOCIE 2014, 2015; the University of Wisconsin Alumni Research Foundation; the Institute of Geophysics, Planetary Physics, and Signatures at Los Alamos National Laboratory; Polish Science Centre grant, DEC-2017/27/B/ST9/02272; Coordinación de la Investigación Científica de la Universidad Michoacana; Royal Society - Newton Advanced Fellowship 180385; Generalitat Valenciana, grant CIDEGENT/2018/034; Chulalongkorn University's CUniverse (CUAASC) grant; Coordinación General Académica e Innovación (CGAI-UdeG), PRODEP-SEP UDG-CA499; Institute of Cosmic Ray Research (ICRR), University of Tokyo, H.F. acknowledges support by NASA under award number 80GSFC21M0002. We also acknowledge the significant contributions over many years of Stefan Westerhoff, Gaurang Yodh and Arnulfo Zepeda Dominguez, all deceased members of the HAWC collaboration. Thanks to Scott Delay, Luciano Díaz and Eduardo Murrieta for technical support.

\section{References}

[1] A.A. Abdo, B. Allen, D. Berley, S. Casanova, C. Chen, D.G. Coyne et al., TeV Gamma-Ray Sources from a Survey of the Galactic Plane with Milagro, Astrophysical Journal Letters 664 (2007) L91 [0705 . 0707].

[2] F. Aharonian, A.G. Akhperjanian, G. Anton, U. Barres de Almeida, A.R. Bazer-Bachi, Y. Becherini et al., Detection of very high energy radiation from HESS J1908+063 confirms the Milagro unidentified source MGRO J1908+06, Astronomy and Astrophysics 499 (2009) 723 [0904 . 3409].

[3] B. Bartoli, P. Bernardini, X.J. Bi, C. Bleve, I. Bolognino, P. Branchini et al., Observation of the TeV Gamma-Ray Source MGRO J1908+06 with ARGO-YBJ, The Astrophysical Journal 760 (2012) 110 [1207 . 6280].

[4] E. Aliu, S. Archambault, T. Aune, B. Behera, M. Beilicke, W. Benbow et al., Investigating the TeV Morphology of MGRO J1908+06 with VERITAS, The Astrophysical Journal 787 (2014) 166 [1404.7185]. 
[5] A. Albert, R. Alfaro, C. Alvarez, J.R.A. Camacho, J.C. Arteaga-Velázquez, K.P. Arunbabu et al., 3HWC: The Third HAWC Catalog of Very-high-energy Gamma-Ray Sources, The Astrophysical Journal 905 (2020) 76 [2007.08582].

[6] A. Abeysekara, A. Albert, R. Alfaro et al., Multiple Galactic Sources with Emission Above $56 \mathrm{TeV}$ Detected by HAWC, Phys. Rev. Lett. 124 (2020) 021102 [1909. 08609].

[7] A. Albert, R. Alfaro, C. Alvarez, J.R. Angeles Camacho, J.C. Arteaga-Velázquez, K.P. Arunbabu et al., Constraints on Lorentz Invariance Violation from HAWC Observations of Gamma Rays above 100 TeV, Physical Review Letters 124 (2020) 131101 [1911.08070].

[8] A.A. Abdo, M. Ackermann, M. Ajello, L. Baldini, J. Ballet, G. Barbiellini et al., PSR J1907+0602: A Radio-Faint Gamma-Ray Pulsar Powering a Bright TeV Pulsar Wind Nebula, The Astrophysical Journal 711 (2010) 64 [1001.0792].

[9] L. Duvidovich, A. Petriella and E. Giacani, Radio study of the extended TeV source VER J1907+062, Monthly Notices of the Royal Astronomical Society 491 (2020) 5732 [1912.13352].

[10] J. Li, R.-Y. Liu, E. de Oña Wilhelmi, D.F. Torres, Q.-C. Liu, M. Kerr et al., Investigating the nature of MGRO j190806 with multiwavelength observations, The Astrophysical Journal Letters 913 (2021) L33.

[11] M.G. Aartsen, M. Ackermann, J. Adams, J.A. Aguilar, M. Ahlers, M. Ahrens et al., Search for steady point-like sources in the astrophysical muon neutrino flux with 8 years of IceCube data, European Physical Journal C 79 (2019) 234.

[12] A.J. Smith, HAWC: Design, Operation, Reconstruction and Analysis, Proceedings of Science (34th International Cosmic Ray Conference) 966 (2015) .

[13] A.U. Abeysekara, A. Albert, R. Alfaro, C. Alvarez, J.D. Álvarez, J.R.A. Camacho et al., Measurement of the Crab Nebula Spectrum Past $100 \mathrm{TeV}$ with HAWC, The Astrophysical Journal 881 (2019) 134 [1905.12518].

[14] A.U. Abeysekara, A. Albert, R. Alfaro, C. Alvarez, J.D. Álvarez, R. Arceo et al., Very-high-energy particle acceleration powered by the jets of the microquasar SS 433, Nature 562 (2018) 82.

[15] G. Vianello, R.J. Lauer, P. Younk, L. Tibaldo, J.M. Burgess, H. Ayala et al., The Multi-Mission Maximum Likelihood framework (3ML), arXiv e-prints (2015) arXiv: 1507.08343 [1507.08343].

[16] A.U. Abeysekara, A. Albert, R. Alfaro, C. Alvarez, J.D. Álvarez, R. Arceo et al., Extended gamma-ray sources around pulsars constrain the origin of the positron flux at Earth, Science 358 (2017) 911 [1711.06223].

[17] K. Malone, A search for spectral hardening in HAWC sources above $56 \mathrm{TeV}$, Proceedings of Science ICRC 2021 (these proceedings) (2021). 
[18] Z. Cao, F. Aharonian, Q. An, Axikegu, L. Bai, Y. Bai et al., Ultrahigh-energy photons up to 1.4 petaelectronvolts frm 12 gamma-ray galactic sources, Nature Astronomy 594 (2021) 33.

[19] C.A. Kierans, AMEGO: exploring the extreme multimessenger universe, in Society of Photo-Optical Instrumentation Engineers (SPIE) Conference Series, 2020.

[20] M.C. Gonzalez-Garcia, F. Halzen and S. Mohapatra, Identifying Galactic PeVatrons with neutrinos, Astroparticle Physics 31 (2009) 437 [0902 . 1176].

[21] F. Halzen, A. Kheirandish and V. Niro, Prospects for detecting galactic sources of cosmic neutrinos with IceCube: An update, Astroparticle Physics 86 (2017) 46 [1609.03072]. 


\section{Full Authors List: HAWC Collaboration}

A.U. Abeysekara ${ }^{48}$, A. Albert ${ }^{21}$, R. Alfaro ${ }^{14}$, C. Alvarez $^{41}$, J.D. Álvarez ${ }^{40}$, J.R. Angeles Camacho ${ }^{14}$, J.C. Arteaga-Velázquez ${ }^{40}$, K. P. Arunbabu $^{17}$, D. Avila Rojas ${ }^{14}$, H.A. Ayala Solares ${ }^{28}$, R. Babu ${ }^{25}$, V. Baghmanyan ${ }^{15}$, A.S. Barber ${ }^{48}$, J. Becerra Gonzalez ${ }^{11}$, E. BelmontMoreno $^{14}$, S.Y. BenZvi ${ }^{29}$, D. Berley ${ }^{39}$, C. Brisbois ${ }^{39}$, K.S. Caballero-Mora ${ }^{41}$, T. Capistrán ${ }^{12}$, A. Carramiñana ${ }^{18}$, S. Casanova ${ }^{15}$, O. Chaparro-Amaro $^{3}$, U. Cotti ${ }^{40}$, J. Cotzomi $^{8}$, S. Coutiño de León ${ }^{18}$, E. De la Fuente ${ }^{46}$, C. de León ${ }^{40}$, L. Diaz-Cruz ${ }^{8}$, R. Diaz Hernandez ${ }^{18}$, J.C. Díaz-Vélez ${ }^{46}$, B.L. Dingus ${ }^{21}$, M. Durocher ${ }^{21}$, M.A. DuVernois ${ }^{45}$, R.W. Ellsworth ${ }^{39}$, K. Engel ${ }^{39}$, C. Espinoza ${ }^{14}$, K.L. Fan ${ }^{39}$, K. Fang $^{45}$, M. Fernández Alonso ${ }^{28}$, B. Fick ${ }^{25}$, H. Fleischhack ${ }^{51,11,52}$, J.L. Flores ${ }^{46}$, N.I. Fraija ${ }^{12}$, D. Garcia ${ }^{4}$, J.A. García-González ${ }^{20}$, J. L. García-Luna ${ }^{46}$, G. García-Torales ${ }^{46}$, F. Garfias ${ }^{12}$, G. Giacinti ${ }^{22}$, H. Goksu ${ }^{22}$, M.M. González ${ }^{12}$, J.A. Goodman ${ }^{39}$, J.P. Harding ${ }^{21}$, S. Hernandez ${ }^{14}$, I. Herzog ${ }^{25}$, J. Hinton ${ }^{22}$, B. Hona ${ }^{48}$, D. Huang ${ }^{25}$, F. Hueyotl-Zahuantitla ${ }^{41}$, C.M. Hui ${ }^{23}$, B. Humensky ${ }^{39}$, P. Hüntemeyer ${ }^{25}$, A. Iriarte ${ }^{12}$, A. Jardin-Blicq ${ }^{22,49,50}$, H. Jhee ${ }^{43}$, V. Joshi ${ }^{7}$, D. Kieda ${ }^{48}$, G J. Kunde ${ }^{21}$, S. Kunwar ${ }^{22}$, A. Lara ${ }^{17}$, J. Lee ${ }^{43}$, W.H. Lee ${ }^{12}$, D. Lennarz ${ }^{9}$, H. León Vargas ${ }^{14}$, J. Linnemann ${ }^{24}$, A.L. Longinotti ${ }^{12}$, R. López-Coto ${ }^{19}$, G. Luis-Raya ${ }^{44}$, J. Lundeen ${ }^{24}$, K. Malone ${ }^{21}$, V. Marandon $^{22}$, O. Martinez ${ }^{8}$, I. Martinez-Castellanos ${ }^{39}$, H. Martínez-Huerta ${ }^{38}$, J. Martínez-Castro ${ }^{3}$, J.A.J. Matthews ${ }^{42}$, J. McEnery ${ }^{11}$, P. Miranda-Romagnoli $^{34}$, J.A. Morales-Soto ${ }^{40}$, E. Moreno ${ }^{8}$, M. Mostafá ${ }^{28}$, A. Nayerhoda ${ }^{15}$, L. Nellen ${ }^{13}$, M. Newbold ${ }^{48}$, M.U. Nisa ${ }^{24}$, R. Noriega-Papaqui ${ }^{34}$, L. Olivera-Nieto ${ }^{22}$, N. Omodei ${ }^{32}$, A. Peisker ${ }^{24}$, Y. Pérez Araujo ${ }^{12}$, E.G. Pérez-Pérez ${ }^{44}$, C.D. Rho ${ }^{43}$, C. Rivière ${ }^{39}$, D. Rosa-Gonzalez ${ }^{18}$, E. Ruiz-Velasco ${ }^{22}$, J. Ryan ${ }^{26}$, H. Salazar ${ }^{8}$, F. Salesa Greus ${ }^{15,53}$, A. Sandoval ${ }^{14}$, M. Schneider ${ }^{39}$, H. Schoorlemmer ${ }^{22}$, J. Serna-Franco ${ }^{14}$, G. Sinnis ${ }^{21}$, A.J. Smith ${ }^{39}$, R.W. Springer ${ }^{48}$, P. Surajbali ${ }^{22}$, I. Taboada ${ }^{9}$, M. Tanner ${ }^{28}$, K. Tollefson ${ }^{24}$, I. Torres ${ }^{18}$, R. Torres-Escobedo $^{30}$, R. Turner ${ }^{25}$, F. Ureña-Mena ${ }^{18}$, L. Villaseñor ${ }^{8}$, X. Wang ${ }^{25}$, I.J. Watson ${ }^{43}$, T. Weisgarber ${ }^{45}$, F. Werner ${ }^{22}$, E. Willox ${ }^{39}$, J. Wood ${ }^{23}$, G.B. Yodh ${ }^{35}$, A. Zepeda ${ }^{4}$, H. Zhou ${ }^{30}$

${ }^{1}$ Barnard College, New York, NY, USA, ${ }^{2}$ Department of Chemistry and Physics, California University of Pennsylvania, California, PA, USA, ${ }^{3}$ Centro de Investigación en Computación, Instituto Politécnico Nacional, Ciudad de México, México, ${ }^{4}$ Physics Department, Centro de Investigación y de Estudios Avanzados del IPN, Ciudad de México, México, ${ }^{5}$ Colorado State University, Physics Dept., Fort Collins, CO, USA, ${ }^{6}$ DCI-UDG, Leon, Gto, México, ${ }^{7}$ Erlangen Centre for Astroparticle Physics, Friedrich Alexander Universität, Erlangen, BY, Germany, ${ }^{8}$ Facultad de Ciencias Físico Matemáticas, Benemérita Universidad Autónoma de Puebla, Puebla, México, ${ }^{9}$ School of Physics and Center for Relativistic Astrophysics, Georgia Institute of Technology, Atlanta, GA, USA, ${ }^{10}$ School of Physics Astronomy and Computational Sciences, George Mason University, Fairfax, VA, USA, ${ }^{11}$ NASA Goddard Space Flight Center, Greenbelt, MD, USA, ${ }^{12}$ Instituto de Astronomía, Universidad Nacional Autónoma de México, Ciudad de México, México, ${ }^{13}$ Instituto de Ciencias Nucleares, Universidad Nacional Autónoma de México, Ciudad de México, México, ${ }^{14}$ Instituto de Física, Universidad Nacional Autónoma de México, Ciudad de México, México, ${ }^{15}$ Institute of Nuclear Physics, Polish Academy of Sciences, Krakow, Poland, ${ }^{16}$ Instituto de Física de São Carlos, Universidade de São Paulo, São Carlos, SP, Brasil, ${ }^{17}$ Instituto de Geofísica, Universidad Nacional Autónoma de México, Ciudad de México, México, ${ }^{18}$ Instituto Nacional de Astrofísica, Óptica y Electrónica, Tonantzintla, Puebla, México, ${ }^{19}$ INFN Padova, Padova, Italy, ${ }^{20}$ Tecnologico de Monterrey, Escuela de Ingeniería y Ciencias, Ave. Eugenio Garza Sada 2501, Monterrey, N.L., 64849, México, ${ }^{21}$ Physics Division, Los Alamos National Laboratory, Los Alamos, NM, USA, ${ }^{22}$ Max-Planck Institute for Nuclear Physics, Heidelberg, Germany, ${ }^{23}$ NASA Marshall Space Flight Center, Astrophysics Office, Huntsville, AL, USA, ${ }^{24}$ Department of Physics and Astronomy, Michigan State University, East Lansing, MI, USA, ${ }^{25}$ Department of Physics, Michigan Technological University, Houghton, MI, USA, ${ }^{26}$ Space Science Center, University of New Hampshire, Durham, NH, USA, ${ }^{27}$ The Ohio State University at Lima, Lima, OH, USA, ${ }^{28}$ Department of Physics, Pennsylvania State University, University Park, PA, USA, ${ }^{29}$ Department of Physics and Astronomy, University of Rochester, Rochester, NY, USA, ${ }^{30}$ Tsung-Dao Lee Institute and School of Physics and Astronomy, Shanghai Jiao Tong University, Shanghai, China, ${ }^{31}$ Sungkyunkwan University, Gyeonggi, Rep. of Korea, ${ }^{32}$ Stanford University, Stanford, CA, USA, ${ }^{33}$ Department of Physics and Astronomy, University of Alabama, Tuscaloosa, AL, USA, ${ }^{34}$ Universidad Autónoma del Estado de Hidalgo, Pachuca, Hgo., México, ${ }^{35}$ Department of Physics and Astronomy, University of California, Irvine, Irvine, CA, USA, ${ }^{36}$ Santa Cruz Institute for Particle Physics, University of California, Santa Cruz, Santa Cruz, CA, USA, ${ }^{37}$ Universidad de Costa Rica, San José, Costa Rica, ${ }^{38}$ Department of Physics and Mathematics, Universidad de Monterrey, San Pedro Garza García, N.L., México, ${ }^{39}$ Department of Physics, University of Maryland, College Park, MD, USA, ${ }^{40}$ Instituto de Física y Matemáticas, Universidad Michoacana de San Nicolás de Hidalgo, Morelia, Michoacán, México, ${ }^{41}$ FCFM-MCTP, Universidad Autónoma de Chiapas, Tuxtla Gutiérrez, Chiapas, México, ${ }^{42}$ Department of Physics and Astronomy, University of New Mexico, Albuquerque, NM, USA, ${ }^{43}$ University of Seoul, Seoul, Rep. of Korea, ${ }^{44}$ Universidad Politécnica de Pachuca, Pachuca, Hgo, México, ${ }^{45}$ Department of Physics, University of Wisconsin-Madison, Madison, WI, USA, ${ }^{46}$ CUCEI, CUCEA, Universidad de Guadalajara, Guadalajara, Jalisco, México, ${ }^{47}$ Universität Würzburg, Institute for Theoretical Physics and Astrophysics, Würzburg, Germany, ${ }^{48}$ Department of Physics and Astronomy, University of Utah, Salt Lake City, UT, USA, ${ }^{49}$ Department of Physics, Faculty of Science, Chulalongkorn University, Pathumwan, Bangkok 10330, Thailand, ${ }^{50}$ National Astronomical Research Institute of Thailand (Public Organization), Don Kaeo, MaeRim, Chiang Mai 50180, Thailand, ${ }^{51}$ Department of Physics, Catholic University of America, Washington, DC, USA, ${ }^{52}$ Center for Research and Exploration in Space Science and Technology, NASA/GSFC, Greenbelt, MD, USA, ${ }^{53}$ Instituto de Física Corpuscular, CSIC, Universitat de València, Paterna, Valencia, Spain 